\title{
The Online Laboratory: Conducting Experiments in a Real Labor Market*
}

\author{
John J. Horton ${ }^{\dagger} \quad$ David G. Rand ${ }^{\ddagger} \quad$ Richard J. Zeckhauser ${ }^{\S}$
}

\begin{abstract}
Online labor markets have great potential as platforms for conducting experiments, as they provide immediate access to a large and diverse subject pool and allow researchers to conduct randomized controlled trials. We argue that online experiments can be just as validboth internally and externally — as laboratory and field experiments, while requiring far less money and time to design and to conduct. In this paper, we first describe the benefits of conducting experiments in online labor markets; we then use one such market to replicate three classic experiments and confirm their results. We confirm that subjects (1) reverse decisions in response to how a decision-problem is framed, (2) have pro-social preferences (value payoffs to others positively), and (3) respond to priming by altering their choices. We also conduct a labor supply field experiment in which we confirm that workers have upward sloping labor supply curves. In addition to reporting these results, we discuss the unique threats to validity in an online setting and propose methods for coping with these threats. We also discuss the external validity of results from online domains and explain why online results can have external validity equal to or even better than that of traditional methods, depending on the research question. We conclude with our views on the potential role that online experiments can play within the social sciences, and then recommend software development priorities and best practices.
\end{abstract}

JEL: J2, C93, C91, C92, C70

Keywords: Experimentation, Online Labor Markets, Internet

\section{Introduction}

Some of the first experiments in economics were conducted in the late 1940s to test predictions from the emerging field of game theory. While the research questions were complex, the tools were

${ }^{*}$ Thanks to Alex Breinin and Xiaoqi Zhu for excellent research assistance. Thanks to Samuel Arbesman, Dana Chandler, Anna Dreber, Rezwan Haque, Robin Yerkes Horton, and Stephanie Hurder for helpful comments, as well as to participants in the Online Experimentation Workshop hosted by Harvard's Berkman Center for Internet and Society. This research has been supported by the NSF-IGERT program "Multidisciplinary Program in Inequality and Social Policy" at Harvard University (Grant No. 0333403).

${ }^{\dagger}$ Harvard University.http://sites.google.com/site/johnjosephhorton.

$\ddagger$ Harvard University.http://www.people.fas.harvard.edu/〜 drand.

${ }^{\S}$ Harvard University.http://www.hks.harvard.edu/fs/rzeckhau. 
simple; paper, pencils, blackboards and marbles were sufficient instruments to present stimuli and capture subjects' choices and actions. ${ }^{1}$ By the early 1990s, researchers had developed tools for conducting experiments over local computer networks, with subjects receiving stimuli and making decisions via computer terminal (Fischbacher, 2007). This development made it easier to carry out game play and collect data, easing the burden placed on experimenters. However, the advantages of computer-mediation were not merely logistical; experimenters also gained greater control over the flow of information and thereby reduced the relevance of potentially confounding factors. Computermediation quickly became the primary means for conducting laboratory experiments. Nevertheless, even today, human subjects are still brought into physical labs to participate in experiments that could, at least in principle, be conducted over the Internet with less inconvenience to the subjects and less cost in time and money to the experimenters. Why have we not built the online laboratory?

The case for online experimentation is strong; the clearest advantage of the online laboratory is that there is no need to physically aggregate subjects and compensate them for their travel. This advantage is so obvious that social scientists recognized it more than a decade ago. In 1997, the National Science Foundation sponsored a workshop called NetLab to investigate the potential of online experimentation (Bainbridge, 2007). That workshop's report identified the major advantages of online experimentation, including larger sample sizes, greater subject diversity, and lengthier experiments. NetLab's participants optimistically concluded:

If the nascent laboratory experimental approach is encouraged and is coupled with new technological innovations, then the SBE [social, behavioral, and economic sciences] disciplines will be primed for major scientific advances.

The "if" in that conclusion hinged on some rather mundane obstacles: funding, particularly for software development, and technical training. Thirteen years have passed; and yet, despite an explosion in the size, usage, and capabilities of the Internet, online experiments are still relatively rare, particularly in economics. During the same period, both field and traditional laboratory experiments have become far more common (Levitt and List, 2009). We believe that the practical problems of

\footnotetext{
${ }^{1}$ For an historical perspective on early experimentation in economics, see Kagel et al. (1995).
} 
(1) recruiting subjects and paying them securely and (2) assuring internal validity - and not those of funding or training constraints - have limited the development of online experimentation.

In this paper, we argue that a recent development, the emergence of online labor markets, effectively and efficiently addresses both the recruitment/payment problem and the internal validity problem. Online labor markets also possess distinct advantages over both laboratory and traditional field experiments. These markets allow workers from around the world to perform tasks amenable to remote completion, such as data entry, computer programming, graphic design and clerical work (Frei, 2009). These markets, although designed for other purposes, make it possible to recruit large numbers of subjects who are ready and able to participate in experiments. The subjects themselves are much more diverse and much less experiment-savvy than subjects in traditional laboratories. Even better, online labor markets are ideal for "experimenter-as-employer" experiments, which overcome some of the artificiality critiques of conventional laboratory experiments, which have the subjects work at contrived tasks and with full knowledge of the experimental nature of their "jobs."

While greater subject diversity and market context are attractive features, the prime advantage of online labor markets is that they permit the level of control needed for causal inference. The creators of these online labor markets - for their own, unrelated purposes - have made it easy to make individual-specific payments, screen out users who do not have valid accounts with the market and prevent workers/subjects from communicating with each other. Researchers can use these features to conduct internally valid experiments online. Furthermore, for certain kinds of research questions in economics, field experiments conducted in online labor markets can have a very high level of external validity - a point we will discuss in depth.

Despite the benefits they offer, online experiments raise issues not frequently encountered in either the laboratory or the field. Just as television shows are not filmed plays, online experiments are not simply laboratory experiments conducted online. This paper discusses these differences and pays close attention to the unique challenges of online experimentation. Despite the caveats and pitfalls, the proof of the value of online experiments is in our replications; we quickly, cheaply, and 
easily reproduce a handful of experimental results known to have external validity. Although we will discuss why online experiments work, the key point is that they do work, at least in the cases we have selected for replication.

In Section 2 we elaborate on our claim that online experiments overcome the classic challenges to causal inference. To back up these claims, we successfully reproduce a series of classic experimental results in Section 3. While these confirmations support our argument, they are not sufficient to establish the validity of online methods. For this reason, in Section 4 we highlight the classic threats to valid causal inference and discuss how they can be overcome in an online setting. In Section 5 we then discuss the external validity of online experimental results. That examination leads naturally to Section 6, in which we discuss the pros and cons of different experimental designs, such as the experimenter-as-employer and traditional laboratory economic games. In addition to creating exciting opportunities for research, online experiments also pose some ethical challenges, which we address in Section 7 . We conclude in Section 8 with our thoughts on the future of the online laboratory.

\section{Online Labor Markets and Experimentation}

At present, the most useful online labor markets, from an experimentation standpoint, are "allpurpose" labor markets where buyers contract with individual sellers (Horton, 2010b). Some of the larger markets in this category include oDesk, Elance, Guru and Amazon's Mechanical Turk (MTurk). ${ }^{2}$ Each of these sites is potentially amenable to experimentation, but MTurk currently offers the best venue due to its robust application programming interface (API) and pricing structure. Even better, experimenters can tap the deep knowledge and experience of web developers to create practically any interface or environment imaginable. All of the experiments discussed in this paper were conducted on MTurk.

Amazon claims that the MTurk workforce is larger than 100,000, but provides no details about

\footnotetext{
${ }^{2}$ There are other online labor markets, structured more like tournaments or prize-based contests, that are less relevant for experimental purposes.
} 
its exact size or composition. In conducting our own experiments, we have had no difficulty in rapidly recruiting very large samples. If one were to try to build a site for online experimentation "from scratch," assuming that the technical hurdles could be overcome, the biggest challenge - as anyone who has started a lab knows - would be the familiar chicken-and-egg problem of attracting users. Few subjects would register unless many potential experiments were already available, and, absent many subjects, few experimenters would register their experiments. Online labor markets solve the chicken-and-egg problem.

\subsection{The advantages of recruiting from labor markets}

Online labor markets offer the benefits associated with recruiting subjects from an active labor market. In addition to allowing for truly massive samples, online labor markets provide diverse samples of both high- and low-skilled individuals, from a wide range of countries. One especially useful dimension of subject diversity is inexperience with economic games. ${ }^{3}$ Further, by using subjects from less-developed countries, experimenters can create relatively high-stakes games for far less money than would be needed if using subjects from developed countries.

Experimenters are not required to tell subjects hired from online labor markets that they are participating in an experiment. For experiments that use classic economic games, subjects might guess they are in a study of some kind; but for real-effort, market-appropriate tasks, workers are unlikely to suspect the academic nature of their work. This advantage is enormous, as one of the sharpest critiques of the experimental method in economics is the inherent artificiality of randomized controlled trials in which subjects are aware of the experiment.

The subjects' lack of knowledge rules out experimenter effects in which the subjects try to produce the effect they believe the experimenters expect. It also rules out "John Henry" effects, in which subjects exert great effort because they treat the experiment game like a competitive contest. For economics in particular, the fact that subjects recruited from online labor markets are already

\footnotetext{
${ }^{3}$ Participants are probably less experiment-savvy than subjects recruited online, at large and specifically for participation in economic experiments. They are certainly less savvy than typical subjects at university research laboratories.
} 
in work environments is a tremendous advantage, as subjects are already making consequential economic decisions. As a result, they are likely to view any task or game from an economic frame of mind.

Experimenter and John Henry effects arise because the subjects know that the setting is experimental. Related problems arise when the subjects know about other experimental situations. Subjects in a less desirable treatment might be upset by their bad luck, which might affect their behaviors. Cook and Campbell (1979) call this "demoralization." Furthermore, even if subjects remain unaware of an experiment and of the nature of the treatment, agents of the experimenter might affect outcomes by, on their own initiative, trying to equalize outcomes, such as by imitation of other treatments or by compensatory equalization (Cook and Campbell, 1979). In online experiments in which subjects have no knowledge of the treatments received by others, the threat of demoralization is minimal. Additionally, in online settings, the experimenter requires no agents, so the comparisons between treatment and control groups yield untainted and more accurate results.

\subsection{Obtaining control and promoting trust}

Most of the concerns of online market creators mirror those of would-be experimenters. Employers worry that workers with multiple accounts might place phony bids or manipulate the reputation system by leaving phony feedback. Similarly, experimenters worry that a subject with multiple accounts might participate in an experiment multiple times. The creators of online labor markets do not want workers to communicate with each other, as it could lead to collusion. Experimenters also worry about workers discussing the details of experiments with each other and possibly colluding. Finally, both employers and experimenters need ways to pay individuals precise amounts of money as rewards for their actions and decisions.

It is now easy to hire and pay workers within the context of online labor markets, yet still quite difficult to do the same online, but outside of these markets. The problem is not technological. The type and quality of communication - email, instant messenger services, voice-over-IP — do not depend on whether the buyer and seller are working inside or outside the online market, and banks 
have been electronically transferring funds for decades. The problem is that it is difficult to create trust among strangers. Trust is an issue not only for would-be trading partners, but also for wouldbe experimenters. The validity of economics experiments depends heavily upon trust, particularly subjects' trust that the promulgated rules will be followed and that all stated facts about payment, the identities of other subjects, etc., are true. This need for trust is a good reason to embed experiments in online labor markets, because the creators of these markets have already taken a number of steps to foster trust. These efforts make both commerce and valid experimentation possible.

All online labor markets use reputation systems to create lasting, publicly-available reputationsreputations that are sacrificed if either buyers or workers behave unfairly (Resnick et al.,, 2000). The market creators proactively screen out undesired participants by taking steps, such as requiring a bank account or valid credit card, before either buyer or seller is allowed to join. With persons who have been accepted, the market creators actively manage memberships and suspend bad actors, creating a form of virtuous selection not found in traditional markets. One kind of bad actor is the non-human, that is, automated scripts that fraudulently perform "work." To combat this potential problem, all sites require would-be members to pass a CAPTCHA, or "completely automated public Turing test to tell computers and humans apart" (von Ahn et al., 2003). ${ }^{4}$ With proper precautions, it is unlikely that computers would show up as subjects, or that any worker/subject would believe they were playing against a computer.

While the "Turing test" form of trust is important, the mundane but perhaps more critical requirement is that workers/subjects trust that buyers/experimenters will actually follow the rules that they propose. To encourage this form of trust, many of the online labor markets require buyers to place funds in escrow, which prevents buyers from opportunistically refusing to pay after taking delivery of the worker's output (which is often an easy-to-steal informational good). In many

\footnotetext{
${ }^{4}$ At least on MTurk, there is some danger of malicious users writing scripts that automatically accept and complete "Human Intelligence Tasks," or HITs. However, these attempts are trivially easy to detect for anything more complicated than a single yes/no question. Further, in our experience, jobs that allow workers to only complete one unit of work (which is almost always the case with experiments) do not attract the attention of scammers writing scripts.
} 
markets, there is some form of dispute arbitration, which encourages the belief that all parties are acting in the shadows of an institution that could hold them accountable for their actions, further promoting trust. Perhaps unsurprisingly, survey evidence suggests that workers in MTurk believe that their online bosses are as fair as employers in their home countries (Horton, 2010a).

\subsection{Experiments in MTurk}

Online experiments in MTurk are quite simple to run. An advertisement is placed for the experiment via the same framework used to advertise jobs in the online labor market. Depending on Institutional Review Board (IRB) requirements and the nature of the experiment, the advertisement may or may not explicitly state that the task is part of an academic study. In MTurk, subjects may begin work on a job without prior approval from the employer. To make this possible, the experimenter needs to create a website that gives the subjects instructions, records their choices, provides them with information as the game progresses and determines their payoffs. Websites can incorporate a range, from simple surveys made with off-the-shelf software to custom-built, elaborate interfaces with designs limited only by time and resources. Currently, there are a number of projects under development to build a set of tools for common experimental tasks, such as assigning subjects to treatment and control groups.

\section{Experiments in the Online Laboratory}

This section discusses research conducted in the online laboratory - both our own work and that of others. For this paper, we conducted three experiments similar to those normally run in physical laboratories, experiments which have well-known and widely reproduced results. We also conducted a natural field experiment in the sense of the taxonomy proposed by Harrison and List (2004), which looked at labor supply response to manipulations in the offered wage. Our first experiment replicated a classic result in framing shown by Tversky and Kahneman (1981). In accordance with numerous duplications in the laboratory, we found that individuals are risk-averse in the domain of gains, and 
risk-seeking in the domain of losses. Subjects were paid a fixed rate for participating.

Our second experiment had subjects play a traditional prisoner's dilemma game. Consistent with previous work, we found a non-zero level of cooperation, indicating the existence of otherregarding preferences, in which subjects do not act solely out of self-interest. Our third experiment had subjects play the same prisoner's dilemma game, after having been randomly assigned to read different "priming" passages of religious or non-religious text. Here we demonstrated the wellestablished fact that stimuli unrelated to monetary payoffs can nonetheless affect subjects' decisions. In both the second and third experiments, subjects earned individualized payments based on their choices and the choices of other workers with whom they were randomly matched retroactively.

In our final experiment, we placed ourselves in the role of the employer. This experimentalistas-employer research design is perhaps the most exciting development made possible by online labor markets. We recruited subjects to perform a simple transcription of a paragraph-sized piece of text. After performing this initial task, subjects were offered the opportunity to perform an additional transcription task in exchange for a randomly determined wage. As expected, we found that workers' labor supply curves slope upwards.

\subsection{Existing research}

Several studies using online subject pools have recently appeared, with computer scientists leading the way. They have all used MTurk, primarily as a way to conduct user studies and collect data suitable for training machine learning algorithms (Sheng et al., 2008; Kittur et al.,, 2008; Sorokin and Forsyth, 2008). In a paper that bridged computer science and economics, Mason and Watts (2009) showed that, although quality is not affected by price, output declines when wages are lowered.

In economics, Fong and Luttmer (2009) conducted an online experiment to investigate racial bias in charitable donations, using Knowledge Networks instead of an online labor market. Among

the several economics papers that used online labor markets, Chen and Horton (2010) measured how MTurk workers respond to wage cuts. They found that unexplained wage cuts decrease out- 
put, but that when the cuts are justified to workers, the former levels of output are maintained. ${ }^{5}$ In a separate paper using MTurk, Horton and Chilton (2010) explored whether a simple rational model can explain worker output. While they found strong evidence that at least some workers are price-sensitive, they also found that a non-trivial fraction are target earners, that is, people who work to achieve certain income targets rather than responding solely to the offered wage. Chandler and Kapelner (2010) have also conducted a natural field experiment on MTurk. They subtly manipulated the meaning of the task and measured whether that meaning affected uptake and work quality, both overall and conditional upon a worker's home country. Although the results are still preliminary, the paper is inherently interesting in that it demonstrates the kinds of experiments that would be difficult or impossible to do in other settings. In addition to conventional academic papers, a number of researchers are conducting experiments on MTurk and posting results on their blogs. Gabriele Paolacci at the University of Venice writes a blog called "Experimental Turk" which focuses on reproducing results from experimental psychology. ${ }^{6}$

\subsection{Replication: Framing}

Traditional economic models assume that individuals are fully rational in making decisions - that people will always choose the option that maximizes their utility, which is wholly-defined in terms of outcomes. Therefore, decision-making should be consistent, and an individual should make the same choice when faced with equivalent decision problems. However, as the watershed experiment of Tversky and Kahneman (1981) (hereafter "TK") demonstrated, this is not, in fact, the case. TK introduced the concept of "framing": that presenting two numerically equivalent situations with different language can lead to dramatic differences in stated preferences. In our current experiment,

\footnotetext{
${ }^{5}$ There are a number of papers that have used the Internet as a test bed for field experimentation, primarily as a way to study auctions (Resnick et al., 2006; Lucking-Reiley, 2000).

${ }^{6}$ Although blogs are certainly not equivalent to peer-reviewed journals, they do allow academics to quickly communicate results and receive feedback. For example, Rob Miller and Greg Little at the MIT Computer Science and Artificial Intelligence Laboratory (CSAIL) host a blog called "Deneme" that reports the results of experiments using TurKit - a Java library developed by Little and others to perform iterative, complex tasks on MTurk (Little et al., 2009).
} 
we replicated the framing effect demonstrated by TK on MTurk. ${ }^{7}$

In TK's canonical example, subjects read one of two hypothetical scenarios. Half of the subjects were given the following Problem 1:

Imagine that the United States is preparing for the outbreak of an unusual Asian disease, which is expected to kill 600 people. Two alternative programs to combat the disease have been proposed. Assume that the exact scientific estimates of the consequences of the programs are as follows: If Program A is adopted, 200 people will be saved. If Program B is adopted, there is $\frac{1}{3}$ probability that 600 people will be saved and $\frac{2}{3}$ probability that no people will be saved.

Which of the two programs would you favor?

The other half were given Problem 2 in which the setup (the first three sentences) was identical but the programs were framed differently:

If Program A is adopted, 400 people will die. If Program B is adopted, there is $\frac{1}{3}$ probability that nobody will die, and $\frac{2}{3}$ probability that 600 people will die.

The two scenarios are numerically identical, but the subjects responded very differently. TK found that in Problem 1, where the scenario was framed in terms of gains, subjects were risk-averse: $72 \%$ chose the certain Program A over the risky Program B. However, in Problem 2, where the scenario was framed in terms of losses, $78 \%$ of subjects preferred Program B.

Using these same prompts, we recruited 213 subjects to see whether they would reproduce this preference reversal on MTurk. We offered a participation fee of US\$0.40. We randomly assigned subjects to a treatment upon arrival. Consistent with TK's results, we found that the majority of subjects preferred Program A in the domain of gains (N=95: 69\% A, 31\% B), while the opposite was true in the domain of losses ( $\mathrm{N}=118: 41 \%$ A, $59 \%$ B). The framing significantly affected, and in fact reversed, the pattern of preferences stated by the subjects $\left(\chi^{2}\right.$ test, $\left.p<0.001\right)$. Thus, we successfully replicated the principle of framing on MTurk.

\footnotetext{
${ }^{7}$ This is the second replication of this result on MTurk. Gabriele Paolacci also performed this experiment and reported the results on his blog, http://experimentalturk.wordpress.com/2009/11/06/asian-disease.
} 


\subsection{Replication: Social preferences}

Another central theme in experimental economics is the existence of social (or "other-regarding") preferences (Andreoni, 1990; Fehr and Schmidt, 1999). Countless laboratory experiments have demonstrated that many people's behaviors are inconsistent with caring only about their own monetary payoffs. (For a review, see Camerer (2003).) Here we replicated the existence of other-regarding preferences on MTurk.

To assess pro-social behavior on MTurk, 113 subjects were recruited to play an incentivized prisoner's dilemma ("PD"), the canonical game for studying altruistic cooperation (Axelrod and Hamilton, 1981). Each subject was informed that he or she had been randomly assigned to interact with another MTurk worker and that they both would have a choice between two options, A or B. In addition to a $\$ .20$ "show-up fee," subjects were further informed of the following payoff structure (in units of cents):

\begin{tabular}{c|c|c|} 
& A (cooperate) & B (not) \\
\hline A (cooperate) & 120,120 & 40,160 \\
\hline B (not) & 160,40 & 80,80 \\
\hline
\end{tabular}

Here, A represents cooperation, B represents defection, and, regardless of the action of one's partner, playing B maximizes one's payoff. Rational self-interested players should therefore always

select B. Given previous evidence from experiments in the laboratory (Camerer, 2003), however, we predicted that MTurk subjects would demonstrate a level of cooperation significantly greater than 0 in a one-shot PD. Consistent with this prediction, we observe a level of cooperation significantly greater than zero $(55 \% \mathrm{C}$ : sign-rank test, $p<0.001)$ and successfully replicated with MTurk the existence of other-regarding preferences on MTurk.

\subsection{Replication: Priming}

Priming is a third behavioral phenomenon which we demonstrated with MTurk. In priming studies, stimuli unrelated to the decision task (and which do not affect the monetary outcomes) can 
nonetheless significantly alter subjects' behaviors. Priming has attracted a great deal of attention in psychology, and, more recently, in experimental economics (Benjamin et al., 2010a).

To employ MTurk to explore the effects of priming, we recruited 189 subjects to play the same PD game described in the previous section. Subjects were randomly assigned to either the religious prime group $(N=90)$ or a neutral prime group $(N=99)$. The religious prime group read a Christian religious passage about the importance of charity (Mark 10:17-23) before playing the PD. The neutral prime group instead read a passage of equal length describing three species of fish before playing the PD. Following the PD, each subject completed a demographic questionnaire reporting age, gender, country of residence, and religious affiliation. The subjects also indicated whether they had ever had an experience which convinced them of the existence of God. Based on previous results using implicit primes with a non-student subject pool (Shariff and Norenzayan, 2007), we hypothesized that the religious prime would increase cooperation, but only among subjects who believed in God.

The results are portrayed in Figure 1. We analyzed the data using logistic regression with robust standard errors, with PD decision as the dependent variable ( $0=$ defect, $1=$ cooperate $)$, and prime $(0=$ neutral, $1=$ religion $)$ and believer $(0=$ does not believe in God, $1=$ believes in God $)$ as independent variables, along with a prime $\times$ believer interaction term. We also included age, gender $(0=$ female, $1=$ male $)$, country of residence $(0=$ non-U.S., $1=\mathrm{U} . \mathrm{S}$.$) , and religion (0=$ non-Christian, $1=$ Christian $)$ as control variables. Consistent with our prediction, we found no significant main effect of prime $(p=0.169)$ or believer $(p=0.12)$, but a significant positive interaction between the two (coeff $=2.15$, $p=0.001)$. We also found a significant main effect of gender $($ coeff $=0.70, p=0.044)$, indicating that women are more likely to cooperate, but no significant effect of age $(p=0.52)$, country of residence $(p=0.657)$ or religion $(p=0.54)$. We demonstrated that the religious prime significantly increases cooperation in the PD, but only among those who believe in God. These findings are of particular note given the mixed results of previous studies regarding the effectiveness of implicit religious primes for promoting cooperation (Benjamin et al., 2010b). We demonstrate that the principle of priming can be observed with MTurk and that the effectiveness of the prime can vary 


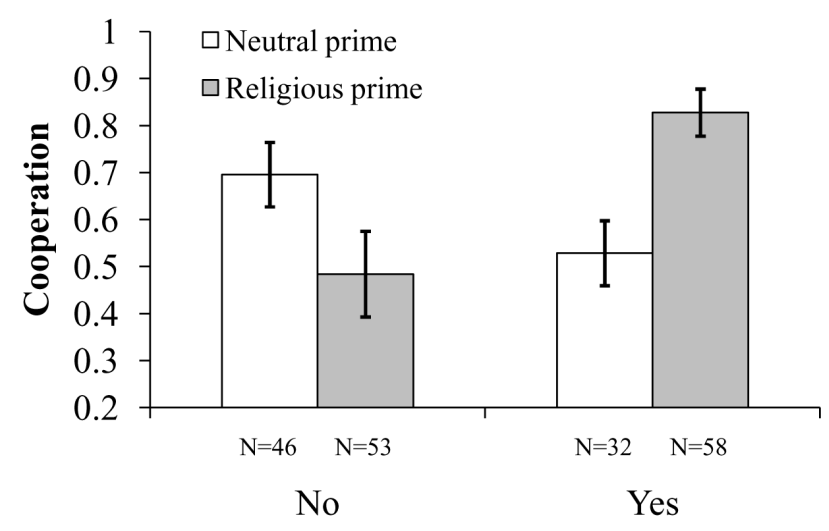

Believe in God

Figure 1: Reading a religious passage significantly increases prisoner's dilemma cooperation among those who believe in God, but not among non-believers.

systematically, depending on the characteristics of the reader.

\subsection{Replication: Labor supply on the extensive margin}

Economic theory predicts that, under most circumstances, increasing the price paid for labor will increase the supply of labor. ${ }^{8}$ In this experiment, we exogenously manipulated the payment offered to different workers and then observed their labor supply. Because the sums involved were so small, we are confident that income effects, at least as traditionally conceived, were inconsequential in this context. We found strong evidence that subjects are more likely to work when wages are high.

When subjects "arrived" at the experiment, we explained that they would answer a series of demographic questions and then perform one paragraph-sized text transcription for a total of 30 cents. They were also told that they would have the opportunity to perform another transcription after the original transcription was completed.

In addition to asking their age, gender and hours per week spent online doing tasks for money, we asked workers to identify their home countries and their primary reasons for participation on MTurk. Because economic opportunities differ by country, we might expect that motivation and

\footnotetext{
${ }^{8}$ The exception is when the increased price changes total wealth to such an extent that changed tastes under the new scenario (i.e., income effects) might be more important than the pure substitution effect.
} 
behavior would also differ by country (Chandler and Kapelner, 2010). Figure 2 presents a mosaic plot showing the cross-tabulation results. We can see that most subjects, regardless of nationality, claimed to be motivated primarily by money. Among those claiming some other motivation, those from India claimed to want to learn new skills, while those from the United States claimed to want to have fun.

For the actual real-effort task, we asked subjects to copy verbatim the text displayed in a scanned image into a separate text box. The text appeared as an image in order to prevent subjects from simply copying and pasting the text into the text box. The advantages of a text transcription task are that it (a) is tedious, (b) requires effort and attention, and (c) has a clearly defined quality measure - namely, the number of errors made by subjects (if the true text is known). We have found it useful to machine-translate the text into some language that is unlikely to be familiar, yet has no characters unavailable on the standard keyboards. Translating increases the error rate by ruling out the use of automated spell-checking, and it prevents subjects from finding the true text somewhere on the web. For this experiment, our text passages were paragraph-sized chunks from Adam Smith's Theory of Moral Sentiments, machine translated into Tagalog, a language of the Philippines.

In our experiment, after completing the survey and the first task, subjects were randomly assigned to one of four treatment groups and offered the chance to perform another transcription for $p$ cents, where $p$ was equal to $1,5,15$ or 25 cents.

As expected, workers receiving higher offers were more likely to accept. Table 1 shows that as the offered price increased, the fraction of subjects accepting the offer rose.

The regression results are $\bar{Y}_{i}=\underbrace{0.0164}_{0.0024} \cdot \Delta p_{i}+\underbrace{0.6051}_{0.0418}$, with $R^{2}=0.13$ and sample size $N=302$, with $\Delta p_{i}=p_{i}-1$. This offsetting transformation makes the regression intercept interpretable as the predicted mean offer uptake when $p=1$. Of course, a linear probability model is false, as it will predict uptake rates greater than 1 . While we could use a general linear model, it makes more sense to tie the problem more closely to our theoretical model of how workers make decisions.

Presumably workers' reservation wages - the minimum amount they are willing to accept to per- 


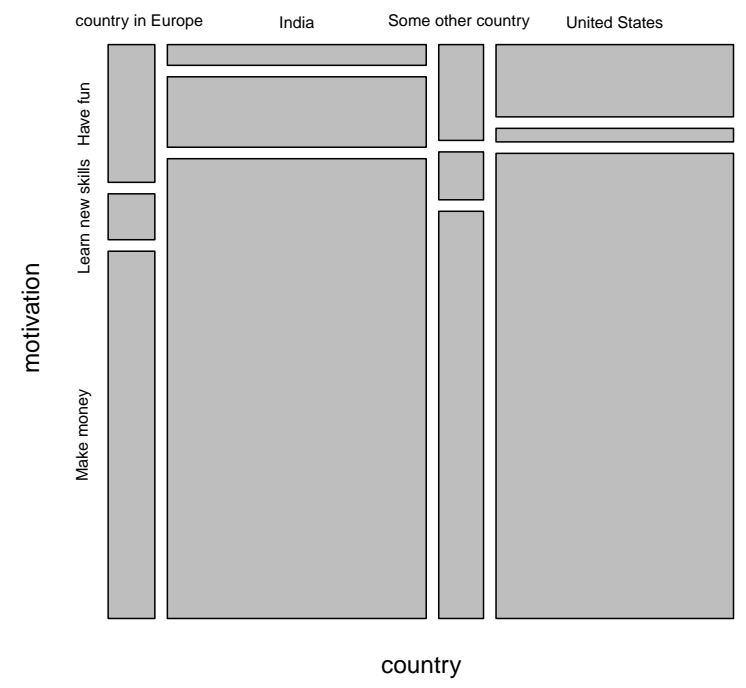

Figure 2: Self-reported motivation for working on Amazon Mechanical Turk (row) cross-tabulated with self-reported country (column) for 302 workers/subjects.

Table 1: Acceptance of paragraph transcription task by offer amount

\begin{tabular}{lccc}
$\begin{array}{l}\text { Amount } \\
\text { (cents) }\end{array}$ & $\begin{array}{c}\text { Offers } \\
\text { Accepted }\end{array}$ & $\begin{array}{c}\text { Offers } \\
\text { Rejected }\end{array}$ & $\begin{array}{c}\text { Percentage } \\
\text { Accepted }\end{array}$ \\
\hline 1 & 34 & 37 & 0.48 \\
5 & 57 & 18 & 0.76 \\
15 & 74 & 5 & 0.94 \\
25 & 71 & 6 & 0.92
\end{tabular}

form some task - have some uknown distribution with cumulative density function cdf $F$. Workers will choose to accept offers to do more work if the offered wages exceed their individual reservation wages. For a task taking $t$ seconds and paying $p_{i}$ cents, then $y_{i}=1 \cdot\left\{p_{i} / t>\omega_{i}\right\}$, where $\omega_{i}$ is the reservation wage. If we assume that $F$ is the log-normal distribution, the distribution parameters that maximize the likelihood of observing our data are $\mu=0.113$ and $\sigma=1.981$. Given the average completion time on the first paragraph, the median reservation wage is US $\$ 0.14 /$ hour. 


\subsection{Summary}

Each of these replication studies was completed on MTurk in less than 48 hours with little effort on our part. The cost was also far less than that of standard lab experiments, at an average cost of less than $\$ 1$ per subject. However, even this low marginal cost vastly understates the comparative efficiency of online experiments. We entirely avoided both the costs associated with hiring full-time assistants and the costs of maintaining a laboratory. We also did not bear the high initial costs of setting up a laboratory.

Low costs would be irrelevant if the results were not informative; yet, despite the low stakes and extreme anonymity of MTurk, the subjects' behavior was qualitatively consistent with findings from the standard laboratory. Our studies demonstrate the power of MTurk to quickly and cheaply give insights into human behavior using both traditional laboratory-style experiments and field experiments.

\section{Internal Validity}

It is reassuring that our experiments achieved results consistent with those of physical laboratories, but we make an independent case for the internal validity of online experiments. Internal validity requires that subjects be appropriately assigned to groups, that selective attrition be ruled out, and that subjects be unable either to interact with or influence one another. These concerns could present challenges in an online setting, where subjects can enter over time and easily drop out of an experiment. If the level of attrition depends on factors such as the nature of the treatment or communications from other subjects, internal validity is jeopardized. Fortunately, proper measures can be taken to overcome, or at least mitigate, these challenges.

\subsection{Unique and independent observations}

MTurk and other online labor markets provide unique observations without much effort on the part of the experimenter, because it is difficult for workers/subjects to have multiple user accounts. 
However, if a worker had multiple accounts, in principle, multiple plays of the experiment would be possible, which could in turn impair the results. At a minimum, the estimated standard errors would be too small. The estimated treatment effect may be biased or even meaningless, since a repeat participant experiences a "treatment" unlike any other and has no obvious control. In contrast, in physical laboratory experiments, multiple plays are easily avoided. If an experiment is conducted in a single session, a person obviously cannot be in two places at once; if the experiment is conducted over multiple sessions, it would be hard for a person both to use a fake ID and not be recognized.

The threat of multiple plays is surely greater online. The anonymity of the Internet rules out the ID approach used in the physical laboratory, and for many experiments, subjects have an incentive to play multiple times. ${ }^{9}$ Fortunately, online labor market creators have strong financial incentives and ample resources to address the problem of multiple accounts and its undesired offspring, multiple plays. Online markets differ in their approaches to the problem of multiple accounts, but all use some combination of terms-of-use agreements and technical approaches. ${ }^{10}$ The important question from the experimental perspective is whether multiple accounts and plays are used sufficiently often in online experiments to threaten their internal validity?

While we admit that multiple accounts are both possible and more probable online, our view is analogous to the view one must take about possible but highly unlikely behaviors that could occur in laboratory or field experiments. For example, some subjects could return with fake IDs or could explain the experiment to others who participate on a different days or at later times. Such behavior is possible, yet it may actually be less likely in the online laboratory because (1) as online labor markets draw on remote and diverse pools, their members are less likely to interact or communicate and (2) the existence of a formal reputation system makes detectably dishonest play costly.

\footnotetext{
${ }^{9}$ Although their per-trial payment would be constant, the marginal cost of completing an experiment would probably decrease (e.g., instructions only have to be read once). If skill were rewarded, then participants would benefit from experience. Even leaving aside declining costs, doing an experiment would buy participants an option: when they found a good deal, they would repeat it.

${ }^{10}$ Some sites require workers to install custom software; presumably this software can detect whether multiple copies of the software are being run on the same computer. Other sites charge membership fees or flat fees for fund transfers, both of which raise the costs of keeping multiple accounts.
} 
Ultimately, the issue of multiple accounts is an empirical question. In our experience to date, we have detected very low numbers of people with multiple accounts, but we have heard anecdotes of a few technically savvy users defeating the system. ${ }^{11}$ Our view is that although multiple accounts surely exist, they are a negligible threat in most online labor markets and are likely to remain so. As in any well-designed regulatory scheme, the steps taken by those who run the sites in online labor markets - and hence in online laboratories - raise the price of prohibited behavior. The goal is to make undesired behavior unlikely, not impossible. Rendering undesired behavior impossible is an unrealistic aspiration.

\subsection{Appropriate assignment}

To be certain that a treatment is having a causal effect on some outcome, we need to assign subjects to treatment and control groups in a way that does not depend on how they will react to a treatment. Randomization accomplishes this goal, but even randomization can, by chance, lead to experimental groups that differ systematically, particularly if there are many explanatory variables. To correct for this problem after randomization we may include pre-treatment variables as regressors. Even better, if we have complete control over assignment, as we do in the online laboratory, we can preemptively avoid the pre-treatment differences problem by employing a blocking design. Such a design identifies the key nuisance factors, creates similar groups on the basis of that factor, and then applies the treatments within each group.

One worker/subject characteristic is arrival time to the experiment. We might expect early and late arrivals to an experiment to differ in systematic ways. For example, immediate respondents are more likely to be heavy users compared to late respondents, and they may respond more immediately to an experiment's advertisement. Time zones introduce a second difference: if an experiment is launched at 1 a.m. PST, experience shows that most of the early respondents (typically in the first 12 hours) will be from Asia and most of the late respondents (in the next 12 hours) will be from the Western hemisphere.

\footnotetext{
${ }^{11}$ We identify them by finding two accounts associated the the same IP address.
} 
In all of our experiments, we stratify according to arrival time. This pairs subjects according to arrival time as closely as possible. Thus even if arrival behavior is related to relevant characteristics, as is likely, this relationship will not bias the composition of the different treatment groups. It is important that subjects be unaware of either the stratification or the randomization; they cannot know what treatment is "next," lest it influence their participation or behavior.

\subsection{Coping with attrition}

Subjects might drop out of the different treatments in an experiment at different rates due to the nature of the treatments. For instance, a video prime that is unpleasant might prompt dropouts that a neutral video would not. Selective attrition leads to selection bias and poses a threat to valid inference in online experiments. The problem is especially acute online because subjects can potentially inspect a treatment before deciding whether to participate. Note that this type of balking is different from potential subjects viewing a description of the overall experiment and deciding not to participate. That is not a concern, as those people are exactly analogous to a those who view an announcement for an experiment but ignore it.

Experiments in a physical laboratory are also subject to attrition disparities, since the participants can get up and leave if they wish. However, it is much easier to quit an experiment on the Internet. Moreover, the time investments required to "try out" an experiment are much lower on the Internet. Thus, if the burden of one treatment is greater than that of another, MTurk subjects are more likely to drop out selectively than their physical laboratory counterparts. The online laboratory has two ways to deal with selective attrition.

The solutions are to collect data on all "arrivals" to an experiment and either, using Method 1, show that attrition is consistent with a random process and assume that attrition is in fact random, or, using Method 2, show that attrition is minimal (that is, close to zero). It is important to note that Method 1 requires the experimenter to establish an empirical fact which is that there is an approximate balance in collected covariates across the groups and to establish an untestable assumption, which is that there is no unobserved sorting that could be driving the results (that is, 
different kinds of subjects are dropping out of the two groups, but by chance the total amount of attrition is the same). When the plausibility of Method 1 is doubtful, Method 2 is superior, though it requires a more elaborate experimental design.

The best way to eliminate attrition is to give subjects strong incentives to continue participating in the experiment after receiving their treatment assignments. In the physical lab, subjects can forfeit their show-up fee by leaving prematurely. Online experiments can do something similar if they employ an initial phase - identical across treatments - that "hooks" subjects into the experiment and ensures that there is no attrition after the hook phase. For example, all subjects might be asked to perform a rather tedious but well-paid transcription task first, before being randomized to the relatively easy treatment and control group tasks. The fee for the transcription task is forfeited if the whole experiment is not completed.

The experimenter then restricts the sample to subjects that persevere through the tedious first phase ex ante. This increases confidence that these subjects will remain for the following phase. In short, this approach has subjects invest themselves in the study in a manner that does not differ across treatment groups. We then raise the price of attrition so that any differences between treatments that might drive non-random attrition are overcome by the incentives to comply. ${ }^{12}$

To use this "hook" strategy ethically, it is important to let subjects know initially some of the details of the experiment. Subjects should always know approximately what they will be doing (with estimates of the time required) and the minimum payment they will receive for doing it. We have found that by providing plenty of information at the outset and by using appropriate hooking tasks, we can consistently drive attrition to zero.

\subsection{Stable unit treatment value assumption}

The stable unit treatment value assumption (SUTVA) requires that any individual's outcome depend only upon his or her treatment assignment, and not upon the treatment assignment or outcome of any other subject (Rubin, 1974). This assumption is violated when subjects can communicate with

\footnotetext{
${ }^{12}$ Physical laboratory experiments essentially create the same pattern of costs, implying incentives not to quit. Much of the effort for participation comes from arranging a schedule and traveling to the lab.
} 
each other about their treatments, choices, or experiences.

Physical laboratory experiments can avoid SUTVA problems by conducting the full experiment in one large session and prohibiting talk during the experiment. In practice, experiments often need to be conducted over several days to get a sufficient sample. Subjects are told not to talk to future prospective subjects, but the extent of compliance with that request is not clear.

The SUTVA problem is both more and less challenging online than in physical laboratories. On the downside, the accumulation of subjects over time is inevitable online. The counterbalancing pluses are that the subjects are widely geographically scattered and less likely to know each other. Furthermore, unlike in laboratory or field experiments, the natural mode of conversations about goings-on in the market take place in publicly viewable discussion forums instead of in private encounters.

While market-specific chat rooms and forums do exist, the creators of online labor markets make it difficult for workers to converse directly with other workers directly in order to prevent "disintermediation" (moving their work offsite to escape fees). In MTurk, workers do not have profiles; in larger markets where workers do have profiles, these do not contain contact information (e.g., name, email, phone number, etc.). In MTurk, "disintermediation" is presumably not as much of a concern, but Amazon is worried that if workers could easily contact each other, they might collude in any number of ways, such as by agreeing to approve fraudulently the work output of their co-conspirators. We recognize, as do the operators of online markets, that some collusion is inevitable. Individuals in a community, including an online community, for example, can choose to participate in the work task and communicate outside the market. But it seems reasonable to expect much less collusion in the online setting than is found among the subjects in lab-based experiments, where many repeat participants are drawn from a concentrated pool of individuals.

On the MTurk discussion boards, workers can and do highlight HITs that they have found particularly interesting or rewarding. Sometimes they discuss the content of the tasks. This could skew the results of an experiment. Fortunately, as an experimentalist, it is easy to monitor these boards and periodically search for mentions of relevant user names or details from the experiment. 
We have been running experiments for over a year, and occasionally search the chat rooms for mention of our user name. So far, it has not appeared in any of these forums. As a practical matter, we advise running experiments quickly, keeping them unremarkable, and periodically checking any associated message boards for discussions of any experimental particulars. ${ }^{13}$ Warning or threatening subjects is not recommended, as this would probably do little to deter collusion; it might instead pique curiosity and prompt discussion.

\section{$5 \quad$ External Validity}

At least two factors affect the external validity of experimental results: (1) its representativeness: how representative the experimental sample is of the population of interest, and (2) its realism: how similar the experimental set-up is to the real world context in which the phenomenon arises. Any experiment, whether in a physical laboratory or online, falls at least a bit short on either factor. External validity is thus a matter of degree, not an absolute.

On the first point, people who choose to participate in social science experiments (or work online) represent a small segment of the population. Subjects in online experiments are also highly selective compared to the U.S. population, though much less selective than the students who make up the subjects in most physical laboratory experiments.

In MTurk, workers from the U.S. are younger, more international, and more likely to be female than the U.S. population, according to self-reported demographics (Ipeirotis, 2010). However, even if subjects "look like" some population of interest in terms of observable characteristics, the selfselection of participation is unavoidable.

On the second point, the decisions made in experiments are usually weak imitations of the real decisions in which we are interested. Real decisions almost always involve higher stakes, greater complexity, and greater decision time, among other differences.

The use of highly selected "convenience samples" is so commonplace in experimental economics

\footnotetext{
${ }^{13}$ It might also be possible to "piggy-back" experiments by working with existing market participants with established, commercial reputations - a attractive option suggested to us by Dana Chandler.
} 
and psychology that it is rarely remarked upon within those fields. However, social scientists from nonexperimental fields, or at least fields with less of an experimental tradition, often object to the use of convenience samples and critique experiments according to both the representativeness of the samples and the realism of the experiments. Their critiques are worth discussing because they are central to the argument that online experiments offer significant value.

We should first consider that the factors of representativeness and realism are relative standardshow similar are the recruited subjects and the experimental contexts to the real phenomena? In any particular experiment, theory determines similiarity. For theories that aspire to be general descriptions of how people make decisions or react to stimuli, any reasonable sample of the population is appropriate. However, theories that cover the behavior of a selected group (for example, young mothers) or behavior in some specialized context (for example, after a disaster) require the sample and the context to match for validity. Because theories in psychology and economics strive for generality, the behavior of a modestly broad group of thinking human beings is usually a reliable guide. For example, if college students homogenize probabilities when making decisions, it is likely that the whole population does so.

To make the point even more sharply, consider the phases of clinical drug trials. In the early stages, for toxicity tests, unselected healthy humans - indeed, in the very early stages, other mammals - make fine subjects. In later stages, for efficacy tests, subjects must suffer from the targeted condition. Toxicity may differ across the population, and it is possible that the target population for some drug might react differently from a random sample, and yet we still feel fairly confident using a convenience sample for toxicity but not for efficacy. The reason is that the questions to be answered - respectively, "Is it safe?" and "Does it work?"-largely determine what kind of sample is needed. 


\subsection{Estimates of changes are reliable:, estimates of levels are much less SO}

Quantitative research in the social sciences generally takes one of two forms: it is either trying to estimate a level or a change. For "levels" research (for example, What is the infant mortality in the United States? Did the economy expand last quarter? How many people support candidate X?), only a representative sample can guarantee a credible answer. For example, if we disproportionately surveyed young people, we could not assess X's overall popularity. For "changes" research (for example, Does mercury cause autism? Do angry individuals take more risks? Do wage reductions reduce output?), the critical concern is the sign of the change's effect. Estimating the magnitude of its effect is secondary. Magnitudes are often assessed in specific populations once a phenomenon has been identified. These two kinds of empirical research often use similar methods and even the same data sources, but one suffers greatly when subject pools are unrepresentative, while the other much less so. Experiments are much more reliable for the analysis of "changes" than for the assessment of "levels."

Laboratory investigations are particularly helpful in "changes" research that seeks to identify phenomena or to elucidate causal mechanisms. Before we even have a well-formed theory to test, we may want to run experiments simply to collect more data on phenomena such as the tendency to confuse nominal and real wages. This kind of research requires an iterative process of generating hypotheses, testing them, examining the data and then honing or discarding hypotheses, followed by more tests, and so on. Because the search space is often large, numerous cycles are needed, which gives the online laboratory an advantage due to its low costs and speedy accretion of subjects. ${ }^{14}$

\footnotetext{
${ }^{14}$ It also increases the danger of "hypothesis mining"- trying out many hypotheses and reporting those that work, quite likely only by chance.
} 


\subsection{Interpreting differences between results from online and physical laboratories}

We have found good agreement between our results and those obtained through traditional means. Nonetheless, there are likely to be measurable differences between results in online and physical laboratory experiments (Eckel and Wilson, 2006). How should one interpret cross-domain differences, assuming they appear to be systematic and reproducible?

First, systematic differences would create puzzles, which can lead to progress. In this sense, the online laboratory can complement the conventional laboratory and conventional laboratory - a point emphasized by Harrison and List (2004). Second, it is not clear that systematic differences should be interpreted as a mark against online experiments. When the Internet was small and few people spent much time online, perhaps it made sense to treat cross-medium differences as an argument against utilizing evidence from online domains. Today, however, people spend a great deal of their time online. ${ }^{15}$ Internet-mediated social interactions are no longer a strange experience familiar only to a tiny fraction of the population.

Suppose that future experiments find that subjects donate less in an online dictator game than in person. ${ }^{16}$ This would tell us something interesting and add to our knowledge: it would not mean that the Internet result is "wrong," assuming that the game is set up properly. The situation is analogous to finding cross-cultural differences in game play (Bohnet et al., 2008; Gneezy et al., 2009). Such results increase our knowledge of the social world; they are not cautionary tales about the need to restrict experimenters to undergraduates at U.S. research universities.

\footnotetext{
${ }^{15}$ A recent New York Times article, "If Your Kids Are Awake, They're Probably Online,"] reported that American kids spend an average of seven to eight hours per day online.

${ }^{16}$ The dictator game is perhaps the simplest game design to measure social preferences: player 1 is given some endowment of money and must decide how much of that money to keep for herself and how much to give to player 2 .
} 


\section{Experimental Designs}

Certain kinds of research designs work well online and are as good as, if not better than, what can be achieved offline. The best example is the experimenter-as-employer natural field experiments, where the experiment in which the interaction is completely unremarkable from the perspective of the workers, who have no idea they are involved in an experiment. Certain kinds of surveys work well online, as we will discuss below. Laboratory-type games are certainly possible, but there are still a number of limitations, some of which will likely be overcome by better software, while others are obviously intractable due to the inherent nature of the Internet. ${ }^{17}$

\subsection{Experimenter-as-employer}

In many field experiments, the researcher participates in the market as an employer, either by creating a new "firm" or by piggy-backing on an existing firm and directing its policies. Workersubjects perform a real-effort task, such as stuffing envelopes, picking fruit, planting trees, etc. The manipulation is usually of such elements as the payment scheme, team composition, or framing. The online environment makes it easy to conduct these kinds of experiments; subjects are simply recruited from the market to perform a task and are randomly assigned to groups. The objective might be to test the role of alternative incentive schemes or to determine how payment affects quality of performance, both central concerns in economics. Depending upon institutional review board requirements, the subjects might not need to be notified that the task is an experiment.

For the actual onine task, it is obviously impossible to have workers perform something physical, yet certain real-effort tasks are well-suited to online completion. One advantage of online work is that it generates remarkably fine details about what workers do at any moment and time, as well as the prevailing context when they made their chices. This kind of very high-frequency data can yield unexpected new insights, as in Bandiera et al. (2009) and Mas and Moretti (2009).

We have found two to be particularly useful: text transcription and the "dot-guessing game"

\footnotetext{
${ }^{17}$ For example, recording physiological responses like eye movement, the galvanic skin response or blood flows to the brain are clearly not possible.
} 
described below. Both provide good-quality metrics and lead to heterogeneous quality, in addition to being culturally neutral. It is also easy to generate new instances of the task (unlike having subjects solve puzzles). At least in the case of text transcription, the task is very similar to the kinds of tasks workers are frequently asked to perform on MTurk, which makes workers less suspicious that they are involved in an experiment.

In the dot-guessing game, subjects examine an image consisting of a very large number of dots, far too many to count feasibly, and are asked to guess the number of dots. This task has a clear error metric, in that it is fully objective (that is, there is a right answer); yet it is similar to a subjective task in that there will be disagreement among subjects in that some subjects will presumably be better estimators than others. Unlike some other tasks used in judgment experiments (for example, estimating the length of the Nile, the grain harvest in Ukraine, etc.), subjects cannot simply look up an answer online, which is an obvious critical difference.

A variant of the experimenter-as-employer paradigm is the paired survey, which is used when one wants to know how some feature of the way a question is posed affects responses. While this kind of experiment is very simple, it can yield powerful conclusions. For example, some of Tversky and Kahneman's classic work, which we replicated, used the paired-survey method to show that people viewed objectively identical scenarios differently depending upon whether an outcome was perceived as a gain or a loss. This simple survey design yielded an insight that has subsequently been shown to be widespread and important. MTurk is ideal for this kind of research.

\subsection{Laboratory-type games online}

Some of the most successful examples of experimental social science use simple interactive games, such as the trust game, the prisoners' dilemma, and market games. Subject interactions are easy to arrange in physical laboratory experiments because all the subjects play at once. In online labor markets, subjects often arrive at the experiment over the course of several hours or days, making subject interactions difficult. There are several solutions to the difficulty presently available and many more under development. 
When subjects are asynchronous, the widely used strategy method (Selten, 1967) — players report what they would do in various hypothetical situations - can identify outcomes in interactive situations. This was the method we employed when performing our own trust-game and ultimatum-game experiments (not yet published). There is some evidence that subjects play "hot" games (those that do not use the strategy method) differently (Brandts and Charness, 2000). Ideally, new software developments will allow for hot interactive games.

If the reliability of the strategy method is accepted, implementation of online experiments is simple. The experimenter needs only to simulate play once all responses have been received. This method also has the advantage of giving more data points. For example, in contrast to a "hot" ultimatum game where we can only observe "accept" or "reject" responses from the second player, in a the "cold" strategy-method game, we can see the subjects' responses to several offers because they must answer the question "Would you accept X?" for several X values.

The online laboratory can allow for "hot" play if there are sufficiently large numbers of respondents who can be matched up as they arrive. Experiments have shown that it is possible to get MTurk workers to wait around for another player. ${ }^{18}$ This kind of approach does require that the experimenter establish some rules for payment if a match cannot be found in a suitable amount of time. Another approach is to pay workers to come back to a website at a certain time to play the game. The great advantage of this method is that it can be used to exactly replicate exactly the current laboratory experience. This method requires web-based interfaces for games. Work at MIT to develop "Seaweed," a web-based experimental platform, represents a strong step in this direction (Chilton et al., 2009). Building such platforms should be a top priority for the experimental social science community.

\section{$7 \quad$ Ethics and Community}

The online laboratory raises new ethical issues. However, it also creates opportunities for setting higher standards of evidence and for fostering greater collaboration among social scientists, both in

\footnotetext{
${ }^{18}$ See this blog post report of the experiment by Lydia Chilton: http://groups.csail.mit.edu/uid/deneme/?p=483
} 
terms of sharing materials and in the adversarial "collaboration" of replication studies, which are now far easier to perform.

\subsection{Ethical implications of moving towards a bench science}

Online experiments can initially propel certain subfields in the social sciences substantially toward "bench science." It is now remarkably easy to design, launch and analyze the results of an experiment. Conducting multiple experiments per week for relatively small amounts of money is now feasible. This is an exciting and welcome development, however, in such an environment, a researcher could turn out a stream of spuriously significant results by burying all that are negative. Even honest researchers can convince themselves of the flaws in their "pilots" and of the legitimacy of the subsequent experiments that happened to yield good results.

Another advantage of online experiments is that they can be run with little assistance from others. This is coupled with the disadvantage of reducing critiques by others of procedures and results. Since there are no lab technicians, no subjects who can be contacted, and no logs on university-run servers, the temptation to cheat may be high. While few researchers would knowingly falsify results, certain professional norms could raise the cost of bad behavior, with the effect of both fostering honesty and dampening skepticism.

The first norm should be machine-readable sharing of experimental materials, as well as detailed instructions on set-up and process. Perhaps some institution, such as a professional organization or the National Science Foundation, could set up a library or clearinghouse for such materials. While most results would probably not be checked by new experiments, requiring all experimenters to make replication very easy would make all results "contestable." This should help make cheating an unprofitable and unpopular strategy. Another advantage of such a norm is that it would reduce costly duplication of programming effort and design. As a current example, the open-source survey software Limesurvey allows researchers to export their survey designs as stand-alone files. Researchers can simply download other people's experimental materials and then deploy their own working versions of surveys/experiments. 
There is a consensus developing in economics to make all data and code publicly available. To adhere to and support this norm is easy in online contexts, but online experimenters should go a step further. Datasets should be publicly available in the rawest form possible (that is, in the format in which the experimental software collected the data), as should the associated code that turned the raw data into the data set. ${ }^{19}$ The Internet makes such sharing a low-cost chore, since the data are invariably generated in machine-readable form.

\subsection{Deception}

There is a well-established ethic in experimental economics against deceiving subjects, an ethic that yields significant positive externalities. Many experiments rely critically on subjects accepting instructions from experimenters at face value. Moreover, deception in money-staked economics experiments could approach and even constitute fraud. The arguments for maintaining this "nodeception" policy in online experiments are even stronger.

Workers in online labor markets are a common resource shared by researchers around the globe. Once experiments reach a significant scale, practicing deception in these markets could pollute this shared resource by creating distrust. In the online world, reputations will be hard to build and maintain, since the experimenter's user name will be the only thing the subject knows about the experimenter. Of course, economists will share the experimental space with psychologists, sociologists and other social scientists who may not share the "no deception" norm. Probably little can be done to police other disciplines, but economists can take steps to highlight to their subjects their adherence to the no-deception rule and to present arguments to others that deception is a threat to the usefulness of these markets. If online experiments become truly widespread, we would expect some sites to prohibit deception, in part because their non-experimenting employers would also be hurt by distrust. Additional forms of certification or enforcement processes are also likely

\footnotetext{
${ }^{19}$ Often it is necessary to clean this data in different ways, such as by dropping bad inputs, or adjusting them to reflect the subject's obvious intent (e.g., if a subject is asked to report in cents and reports .50, it might reasonable to infer they meant 50 cents, not a half-cent). By making all trimming, dropping, reshaping, etc., programmatic, it is easier for other researchers to identify why a replication failed, or what seemingly innocuous steps taken by the original researcher drove the results.
} 
to arise.

\subsection{Software development}

The most helpful development in the short term would be better (and better-documented) tools. There are a number of software tools under active development that should make online experimentation far easier. The first goal should probably be to port some variant of zTree to run on the Internet. The MIT initiative "Seaweed" is a nascent attempt at this goal, but it needs much more development.

The research community should, as much as possible, leverage existing open-source tools. For example, many experiments simply require some kind of survey tool. The previously mentioned open-source project "Limesurvey" already offers an excellent interface, sophisticated tools, and perhaps most importantly, a team of experienced and dedicated developers and a large, non-academic user base. Obviously some tools, such as those for complex games, will have to be custom-built for experiments. Advances in software are required for games calling for simultaneous participation by two or more subjects. Such software is being developed in multiple locales.

\section{Conclusion}

In this paper, we have argued that experiments conducted in online labor markets can be just as valid as other kinds of experiments with the added benefit of greatly reduced cost and inconvenience. In our replication of well-known experiments, we relied on MTurk, as MTurk is currently the best online labor market for experimentation. However, as other online labor markets mature and add their own application programming interfaces, it should be easier to conduct experiments in other domains. It might even be possible to recruit a panel of subjects to participate in a series of experiments. Many other markets have the advantage ove MTurk, whose workers remain anonymous, of offering greater ease in learning about the subjects/workers.

In this paper, we have shown that it is possible to replicate, quickly and inexpensively, findings 
from traditional, physical laboratory experiments in the online laboratory. We have also argued that experiments conducted in the context of online labor markets have internal validity, and that the issue of external validity is theory-dependent as opposed to domain-dependent. The domains where results will and will not have external validity are arbitrary and require judgement by researchers. Lastly, we have proposed a number of new and desirable norms and practices that could enhance the usefulness and credibility of online experimentation. We believe that the future is bright for the online laboratory and predict that, as the NetLab workshop quotation in our introduction suggested, the social sciences are indeed primed for major scientific advances.

\section{References}

Andreoni, J., "Impure Altruism and Donations to Public Goods: A Theory of Warm-glow Giving," The Economic Journal, 1990, pp. 464-477.

Axelrod, R. and WD Hamilton, "The Evolution of Cooperation," Science, 1981, 211 (4489), 1390.

Bainbridge, W.S., "The Scientific Research Potential of Virtual Worlds," Science, 2007, 317 (5837), 472 .

Bandiera, O., I. Barankay, and I. Rasul, "Social Connections and Incentives in the Workplace: Evidence from Personnel Data," Econometrica, 2009, 77 (4), 1047-1094.

Benjamin, D.J., J.J. Choi, and A. Strickland, "Social Identity and Preferences," American Economic Review (forthcoming), 2010.

_, _, and G. Fisher, "Religious Identity and Economic Behavior," Cornell University Mimeo, 2010. 
Bohnet, I., F. Greig, B. Herrmann, and R. Zeckhauser, "Betrayal Aversion: Evidence from Brazil, China, Oman, Switzerland, Turkey, and the United States," American Economic Review, 2008, 98 (1), 294-310.

Brandts, J. and G. Charness, "Hot vs. Cold: Sequential Responses and Preference Stability in Experimental Games," Experimental Economics, 2000, 2 (3), 227-238.

Camerer, C., Behavioral game theory: Experiments in Strategic Interaction, Princeton University Press Princeton, NJ, 2003.

Chandler, D. and A. Kapelner, "Breaking Monotony with Meaning: Motivation in Crowdsourcing Markets," University of Chicago mimeo, 2010.

Chen, D. and J. Horton, "The Wages of Pay Cuts: Evidence from a Field Experiment," Harvard University Mimeo, 2010.

Chilton, L.B., C.T. Sims, M. Goldman, G. Little, and R.C. Miller, "Seaweed: A Web Application for Designing Economic Games," in "Proceedings of the ACM SIGKDD Workshop on Human Computation" ACM 2009, pp. 34-35.

Cook, T.D. and D.T. Campbell, Quasi-Experimentation, Houghton Mifflin Boston, 1979.

Eckel, C.C. and R.K. Wilson, "Internet Cautions: Experimental Games with Internet Partners," Experimental Economics, 2006, 9 (1), 53-66.

Fehr, E. and K.M. Schmidt, "A Theory of Fairness, Competition, and Cooperation," Quarterly Journal of Economics, 1999, 114 (3), 817-868.

Fischbacher, U., "z-Tree: Zurich Toolbox for Ready-Made Economic Experiments," Experimental Economics, 2007, 10 (2), 171-178.

Fong, C.M. and E.F.P. Luttmer, "What Determines Giving to Hurricane Katrina Victims? Experimental Evidence on Racial Group Loyalty," American Economic Journal: Applied Economics, 2009, 1 (2), 64-87. 
Frei, Brent, "Paid Crowdsourcing: Current State \& Progress toward Mainstream Business Use," Produced by Smartsheet.com, 2009.

Gneezy, Uri, K.L. Leonard, and J.A. List, "Gender Differences In Competition: Evidence From A Matrilineal And A Patriarchal Society," Econometrica, 2009, 77 (5), 1637-1664.

Harrison, G.W. and J.A. List, "Field Experiments," Journal of Economic Literature, 2004, 42 (4), 1009-1055.

Horton, J., "The Condition of the Turking class: Are Online Employers Fair and Honest?," Arxiv preprint arXiv:1001.1172, 2010.

_ , "Online Labor Markets," Working paper, Harvard University, 2010.

— and L. Chilton, "The Labor Economics of Paid Crowdsourcing," Proceedings of the 11th ACM Conference on Electronic Commerce (forthcoming), 2010.

Ipeirotis, P., "Demographics of Mechanical Turk," New York University Working Paper, 2010.

Kagel, J.H., A.E. Roth, and J.D. Hey, The Handbook of Experimental Economics, Princeton University Press Princeton, NJ, 1995.

Kittur, A., E.H. Chi, and B. Suh, "Crowdsourcing User Studies with Mechanical Turk," 2008.

Levitt, S.D. and J.A. List, "Field Experiments in Economics: The Past, the Present, and the Future," European Economic Review, 2009, 53 (1), 1-18.

Little, G., L.B. Chilton, M. Goldman, and R.C. Miller, "TurKit: Tools for Iterative Tasks on Mechanical Turk," in "Proceedings of the ACM SIGKDD Workshop on Human Computation" ACM 2009.

Lucking-Reiley, D., "Auctions on the Internet: What's Being Auctioned, and How?," The Journal of Industrial Economics, 2000, 48 (3), 227-252.

Mas, A. and E. Moretti, "Peers at work," American Economic Review, 2009, 99 (1), 112-145. 
Mason, W. and D.J. Watts, "Financial Incentives and the Performance of Crowds," in "Proceedings of the ACM SIGKDD Workshop on Human Computation" ACM 2009, pp. 77-85.

Resnick, P., K. Kuwabara, R. Zeckhauser, and E. Friedman, "Reputation Systems," Communications of the ACM, 2000, 43 (12), 45-48.

_, R. Zeckhauser, J. Swanson, and K. Lockwood, "The Value of Reputation on eBay: A Controlled Experiment," Experimental Economics, 2006, 9 (2), 79-101.

Rubin, D.B., "Estimating Causal Effects of Treatments in Randomized and Nonrandomized Studies," Journal of Educational Psychology, 1974, 66 (5), 688-701.

Selten, R., "Die Strategiemethode zur Erforschung des eingeschrankt rationalen Verhaltens im Rahmen eines Oligopolexperiments," Beitrage zur experimentellen Wirtschaftsforschung, 1967, 1, $136-168$.

Shariff, A.F. and A. Norenzayan, "God is Watching You," Psychological Science, 2007, 18 (9), 803-809.

Sheng, V.S., F. Provost, and P.G. Ipeirotis, "Get another label? Improving Data Quality and Data Mining using Multiple, Noisy Labelers," in "Proceeding of the 14th ACM SIGKDD international conference on Knowledge discovery and data mining" ACM 2008, pp. 614-622.

Sorokin, A. and D. Forsyth, "Utility Data Annotation with Amazon Mechanical Turk," University of Illinois at Urbana-Champaign, Mimeo, 2008, 51, 61820.

Tversky, A. and D. Kahneman, "The Framing of Decisions and the Psychology of Choice," Science, 1981, 211 (4481), 453.

von Ahn, L., M. Blum, N.J. Hopper, and J. Langford, "CAPTCHA: Using Hard AI Problems for Security," Lecture notes in computer science, 2003, pp. 294-311. 